\title{
Structural origin of the pinpoint-composition effect on the glass-forming ability in the NiNb alloy system
}

\author{
Liang Yang, ${ }^{\text {a) }}$ Xiang-fei Meng, and Gu-qing Guo \\ College of Materials Science and Technology, Nanjing University of Aeronautics and Astronautics, \\ Nanjing 210016, People's Republic of China
}

(Received 12 August 2013; accepted 4 October 2013)

\begin{abstract}
Great research efforts to investigate the glass-forming ability (GFA) in alloys have been made, leading to an observation that a pinpoint composition produces the best glass-forming characteristics. The reason for this observation is still unknown, limiting the development of bulk metallic glasses (MGs) with a relatively large size. In this work, systematic experimental measurements coupled with calculations were performed to address this issue using the $\mathrm{NiNb}$ binary alloy system. It is found that the atomic-level packing efficiency and the clusters-level regularity parameters strongly contribute to their GFA. In particular, the best glass former found in a pinpoint composition possesses the local maximum of the atomic-packing efficiency and the highest degree of the cluster regularity. This work provides an understanding of GFA from atomic and cluster levels and will shed light on the development of more MGs with relatively large critical casting sizes.
\end{abstract}

\section{INTRODUCTION}

Understanding the mechanisms of glass formation in alloys has been a long-standing issue since the discovery of the first metallic glass (MG) with the composition of $\mathrm{Au}_{75} \mathrm{Si}_{25}$ in $1960 .{ }^{1}$ So far, various rules, principles, and criteria have been presented to guide the development of amorphous alloys having high glass-forming ability $(\mathrm{GFA})^{2-7}$ because high GFA is required for a broad application of MGs as engineering materials. MGs with high GFA usually can be fabricated in multicomponent alloy systems containing at least three elements. However, recently, MGs with relatively high GFA were successfully prepared in binary alloy systems, such as the $\mathrm{CuZr}^{8}$ and the $\mathrm{NiNb}^{9}$ systems. These simple alloys have attracted intense interest because they have unique properties ${ }^{10-12}$ and they are the representative models for studying glass-forming mechanisms. ${ }^{13-16}$ Further more, studying the binary MGs may enhance our understanding of the formation of their corresponding multicomponent MGs. ${ }^{17-21}$ An interesting phenomenon was found that although these binary systems have the broad composition regions forming MG, the best glass formers are located at some pinpoint compositions. ${ }^{8,9}$ The existing rules and criteria fail to explain this phenomenon, resulting in the absence of the guiding principle for developing more $\mathrm{MG}$ compositions with high GFA in binary alloy systems.

The microstructures of MGs have been largely studied. The explicit or direct structural picture is far from being

\footnotetext{
a) Address all correspondence to this author.

e-mail: yangliang@nuaa.edu.cn

DOI: $10.1557 /$ jmr.2013.318
}

established due to the structural complexity in this kind of glassy materials. Nevertheless, fortunately, many structural models have been proposed by studying the clusters theoretically or experimentally, including the hard-sphere random-packing model, ${ }^{22}$ the stereochemically designed model, ${ }^{23}$ the efficient cluster-packing model, ${ }^{24}$ and the quasi-equivalent cluster model. ${ }^{25}$ These structural models revealed the short-to-medium range orderings in MGs, which are the structural mechanism of the high GFA and unique properties in various MGs. This suggests that the above-mentioned issue might be addressed from the aspect of atomic- and cluster-level structures.

In this work, a feasible scheme to address this issue was presented. A structural model was obtained by simulating the synchrotron radiation-based experimental data, and a series of calculations were performed based on this model. The NiNb binary alloy system was selected as the research prototype because it has: (i) a broad composition region forming MG ribbons ${ }^{26}$; (ii) an identified pinpoint composition $\left(\mathrm{Ni}_{62} \mathrm{Nb}_{38}\right)$ having relatively high $\mathrm{GFA}^{9}$; (iii) a relatively simple system, guaranteeing the reliability in structural research; (iv) some corresponding multicomponent alloys possessing high GFA and unique properties, such as $\mathrm{NiNbZr}^{21} \mathrm{Ni}_{x} \mathrm{Nb}_{100-x}(x=60,61,62,63$, and 64 at. \%) alloys were selected and studied, whose identified GFAs are shown in Table I. In the present work, it reveals that the atomic-level packing efficiencies and the regularity of cluster-level local structures are the short-range structural features significantly contributing to the GFA in MGs. In particular, the best glass formers are located in compositions that the above-mentioned parameters have the local maxima values. 
TABLE I. One indicator of the GFA in alloys is the critical casting size (CCS) of MGs. Thus, CCSs denoting the GFAs corresponding to the selected compositions in the NiNb binary alloy systems are listed below. The data are quoted from Ref. 21.

\begin{tabular}{lc}
\hline \hline Alloy compositions & CCS (mm) \\
\hline $\mathrm{Ni}_{60} \mathrm{Nb}_{40}$ & 1.0 \\
$\mathrm{Ni}_{61} \mathrm{Nb}_{39}$ & 1.5 \\
$\mathrm{Ni}_{62} \mathrm{Nb}_{38}$ & 2.0 \\
$\mathrm{Ni}_{63} \mathrm{Nb}_{37}$ & 1.5 \\
$\mathrm{Ni}_{64} \mathrm{Nb}_{36}$ & N/A \\
\hline \hline
\end{tabular}

In our previous work, the atomic-level packing efficiency and cluster-level regularity factors affecting the GFA in $\mathrm{ZrCu}$ alloys have been supposed. ${ }^{27}$ Nevertheless, more proofs should be provided by studying more binary alloy systems, such as the $\mathrm{NiNb}$. Especially, the differences of the measured structural features between the $\mathrm{NiNb}$ selected compositions are found to be more obvious than those in the corresponding $\mathrm{ZrCu}$ ones, leading to more convincing evidence that the GFA is tuned by the fine atomic-to-cluster level structural factors.

\section{EXPERIMENTAL AND SIMULATION METHODS}

The mixture of $\mathrm{Ni}(99.9 \mathrm{wt} \%)$ and $\mathrm{Nb}(99.9 \mathrm{wt} \%)$ metals was melted to prepare the alloy ingots in Ti-gettered high-purity argon atmosphere, according to the selected $\mathrm{NiNb}$ compositions. These ingots were melted at least 4 times to ensure their compositional homogeneity. The melt spinning method was adopted to prepare the corresponding amorphous ribbons, which have a cross section of $0.04 \times 2 \mathrm{~mm}^{2}$.

The synchrotron radiation-based high-energy (about $100 \mathrm{keV}$ ) $\mathrm{x}$-ray diffraction was performed to measure the structures for all the samples at the beam line, BW5, of Hasylab in Germany. The two-dimensional diffraction data were recorded using a Mar345 image plate. Subsequently, using the transmission mode, $\mathrm{Ni}$ and $\mathrm{Nb} \mathrm{K}$-edge extended $\mathrm{x}$-ray absorption fine structure (EXAFS) measurements were performed at the beam lines, BL14W1, in the Shanghai Synchrotron Radiation Facility of China, and U7C, in the National Synchrotron Radiation Laboratory of China. The diffraction patterns and EXAFS spectra were normalized via a standard data-reduced procedure, ${ }^{28}$ using the software PDFget $\mathrm{X}^{29}$ and Visual Processing in EXAFS Researches (VIPER), ${ }^{30}$ respectively. To obtain the atomic structural information as reliably as possible, both the normalized diffraction and EXAFS data were simulated simultaneously under the framework of reverse Monte-Carlo (RMC). ${ }^{25,31}$ Subsequently, the simulated atomic structural models were further analyzed by the Voronoi tessellation method. ${ }^{31,32}$

The RMC simulation is an iterative method extensively used for building the possible structural models for probing structural information in disordered systems that agree quantitatively with the available experimental data (such as the synchrotron radiation-based x-ray diffraction, EXAFS, and neutron diffraction data). In particular, it is available for glassy alloys. ${ }^{33}$ In this work, the synchrotron radiation-based $\mathrm{x}$-ray diffraction and EXAFS data were adopted and simulated simultaneously in the RMC frame, using the software RMCA. ${ }^{34}$ The initial cubic boxes contain 40,000 random-distributed $\mathrm{Ni}$ and $\mathrm{Nb}$ atoms, matching the selected $\mathrm{Ni}_{x} \mathrm{Nb}_{100-x}(x=60,61,62,63$, and 64 at.\% $)$ compositions. During the RMC simulation, after all the atoms move randomly within a determined time interval, the experimental diffraction and EXAFS data are compared with the simulation spectra using the iterative calculation expression $^{35}$ :

$$
\begin{aligned}
\delta^{2}= & \frac{1}{\varepsilon^{2}} \sum_{n}\left(S_{m}\left(Q_{n}\right)-S_{\exp }\left(Q_{n}\right)\right)^{2} \\
& +\frac{1}{\varepsilon_{\mathrm{Ni}}^{2}} \sum_{n}\left(\chi_{m, \mathrm{Ni}}\left(k_{n}\right)-\chi_{\exp , \mathrm{Ni}}\left(k_{n}\right)\right)^{2} \\
& +\frac{1}{\varepsilon_{\mathrm{Nb}}^{2}} \sum_{n}\left(\chi_{m, \mathrm{Nb}}\left(k_{n}\right)-\chi_{\mathrm{exp}, \mathrm{Nb}}\left(k_{n}\right)\right)^{2}
\end{aligned}
$$

where $\delta^{2}$ denotes the deviation between the experimental and simulation data, the $\varepsilon$ parameters regulate the weight of the data set in the fitting procedure, the $S(Q)$ and $\chi(k)$ parameters are the normalized XRD structural factor and the EXAFS signal, respectively. The subscripts, " $m$ " and "exp" denote the status of simulations and experiments, respectively. When the calculated data match the experimental spectra, the simulation is stopped, and all the atoms are "frozen" with determined three-dimensional positions in the cubic box, and an atomic-structural model is accordingly obtained, which may be available for further analyses.

According to the Voronoi original algorithm, ${ }^{36}$ each convex Voronoi polyhedron (VP) can be built by connecting the perpendicular bisectors between a center atom and all of its neighboring atoms. Each VP is indexed as $<n 3, n 4, n 5, n 6, \ldots>$, where $n i$ denotes the number of $i$-edged faces on the surface of this polyhedron. Each VP is embedded in a corresponding convex Voronoi cluster (VC), which is made up of a solute (center) atom and its neighboring solvent (shell) atoms (In our case, VCs were extracted from the RMC simulated structural model). Thus, $\sum n i$ also denotes the number of the shell atoms in one $\mathrm{VC}$, i.e., the first-shell coordination number $(\mathrm{CN})$ of the center atom. It also requires that all VCs should be closed structural units, which can be accomplished by piling up a set of Delaunay tetrahedrons with the common vertex at the same atom (the center atom). ${ }^{37}$ This means that their surfaces are only made up of triangular faces, i.e., they could be regarded as deltahedra. ${ }^{25}$ 


\section{RESULTS AND DISCUSSION}

Figures 1(a)-1(c) show the two-dimensional x-ray diffraction patterns (we only show the data of $\mathrm{Ni}_{62} \mathrm{Nb}_{38}$ ), the structural factor, $S(Q)$, and the total pair distribution function, $G(r) . S(Q)$ and $G(r)$ curves can be deduced from the two-dimensional diffraction image. The amorphous nature of these five samples can be confirmed because there are no circle lines or dots in the two-dimensional diffraction patterns and no sharp peaks behind the first strong peak in the $S(Q)$ curves, while these features are usually found in the diffraction data of polycrystals or single crystals. ${ }^{38}$ The oscillations of their $S(Q)$ or $G(r)$ data are almost the same, indicating that they possess the similar basic structural features. This also indicates the absence of the direct quantitative observation of the pinpoint-composition effect on the GFAs in these five NiNb samples. Nevertheless, the fine structural difference between these samples can be distinguished by further simulation of the experimental data.

Figures 2(a)-2(d) show the calculated data obtained from the RMC simulation on the synchrotron radiationbased diffraction and EXAFS signals, including: (i) the three-dimensional structural model containing 40,000 position-determined $\mathrm{Ni}$ and $\mathrm{Nb}$ atoms (we only show the data of $\mathrm{Ni}_{62} \mathrm{Nb}_{38}$ ); (ii) to (iv) the simulated $S(Q)$ curves, the $\mathrm{Ni}$ and $\mathrm{Nb} \mathrm{K}$-edge simulated EXAFS signals in the $\mathrm{NiNb}$ alloys, along with their corresponding experimental spectra. The good matching between all the experiment/simulation pairs confirms the success of the RMC simulations. Based on the simulated structural models, the atomic- and cluster-level structural information can then be deduced. ${ }^{39}$

At the atomic scale, the deduced structural information includes the first-shell $\mathrm{CNs}$ and the average atomic pair

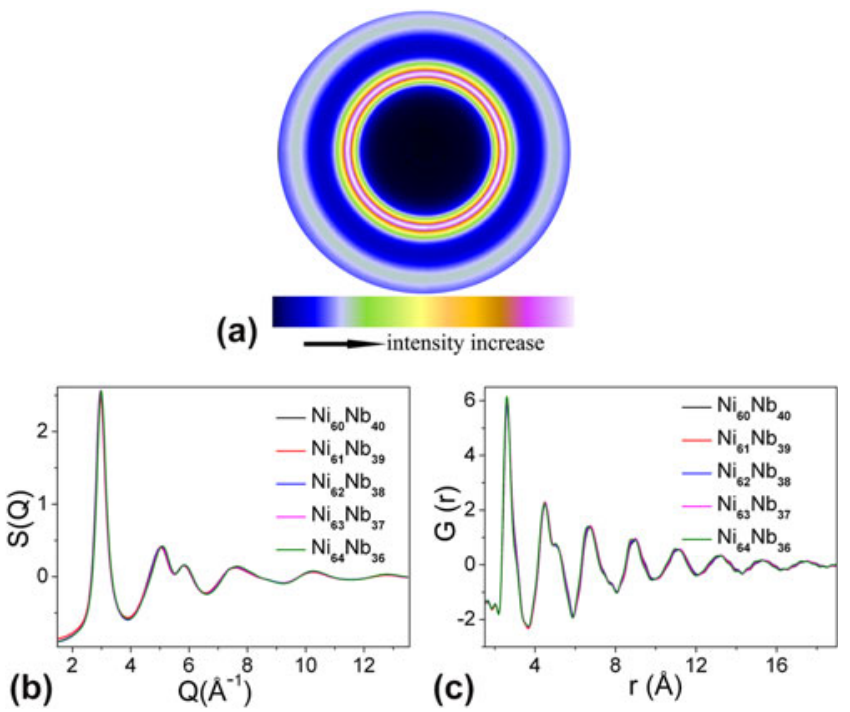

FIG. 1. The synchrotron radiation-based XRD data, including: (a) the two-dimensional diffraction pattern of $\mathrm{Ni}_{62} \mathrm{Nb}_{38}$, (b) the normalized structure factor, $S(Q)$, and (c) the total pair distribution function, $G(r)$, for all five samples. (color online) distances $(R)$, as listed in Table II. There is no obvious difference of the $\mathrm{Ni}-\mathrm{Ni}, \mathrm{Ni}-\mathrm{Nb}$, and $\mathrm{Nb}-\mathrm{Nb} R$ values between $\mathrm{Ni}_{60} \mathrm{Nb}_{40}, \mathrm{Ni}_{61} \mathrm{Nb}_{39}, \mathrm{Ni}_{62} \mathrm{Nb}_{38}, \mathrm{Ni}_{63} \mathrm{Nb}_{37}$, and $\mathrm{Ni}_{64} \mathrm{Nb}_{36}$ samples. The $\mathrm{Ni}-\mathrm{Ni}, \mathrm{Ni}-\mathrm{Nb}, \mathrm{Nb}-\mathrm{Nb}$, or $\mathrm{Nb}-\mathrm{Ni}$ $\mathrm{CN}$ changes monotonically with the $\mathrm{NiNb}$ compositional variation. It seems that there is no unique structural character in the $\mathrm{Ni}_{62} \mathrm{Nb}_{38}$ bulk MG. To deduce more information, the total $\mathrm{CN}$ values around $\mathrm{Ni}$ or $\mathrm{Nb}$ center atoms $(\mathrm{Ni}-\mathrm{M}$ or $\mathrm{Nb}-\mathrm{M}, \mathrm{M}=\mathrm{Ni}+\mathrm{Nb}$ ) were calculated and plotted in Fig. 3.

According to Miracle's efficient cluster-packing model that the $\mathrm{CN}$ around the center atom increases with the radius ratio between the center and the shell atoms,${ }^{40}$ the $\mathrm{CNs}$ of both $\mathrm{Ni}$ and $\mathrm{Nb}$ centers should monotonically increase with the concentration of $\mathrm{Ni}$ because the center/shell radius ratio will increase when more smaller $\mathrm{Ni}$ atoms replace the larger $\mathrm{Nb}$ ones at the shell site (the Goldschmidt atomic radius of $\mathrm{Ni}$ is $1.26 \AA$ while that of $\mathrm{Nb}$ is $1.45 \AA$ ). In our case, the $\mathrm{CNs}$ of $\mathrm{Ni}$ and $\mathrm{Nb}$ centers of $\mathrm{Ni}_{x} \mathrm{Nb}_{100-x}$ monotonically increase when $x$ changes from $60,61,63$, to 64 , which is consistent with Miracle's structural model. However, an abnormal phenomenon can be observed that the CNs of $\mathrm{Ni}$ and $\mathrm{Nb}$ centers in $\mathrm{Ni}_{62} \mathrm{Nb}_{38}$ are even higher than those in $\mathrm{Ni}_{63} \mathrm{Nb}_{37}$, respectively. In other words, the total $\mathrm{CNs}$ around both $\mathrm{Ni}$ and $\mathrm{Nb}$ centers exhibit a local maximum at the $\mathrm{Ni}_{62} \mathrm{Nb}_{38}$ composition, which indicates that atomic-level fine structural difference between $\mathrm{Ni}_{62} \mathrm{Nb}_{38}$ and other four compositions does exist.

So far, clusters with one center atom and some shell atoms have been regarded as the basic building blocks to (a)
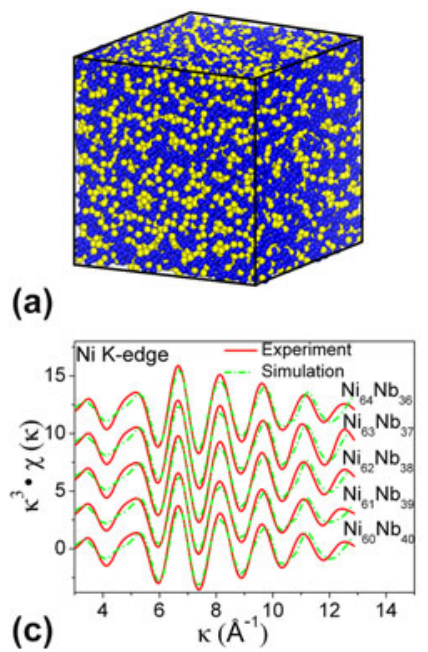

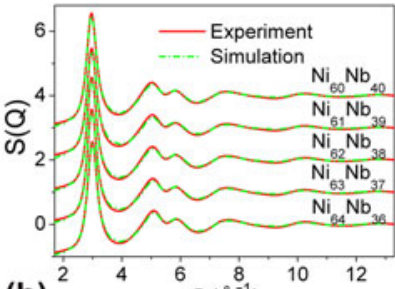

(b)

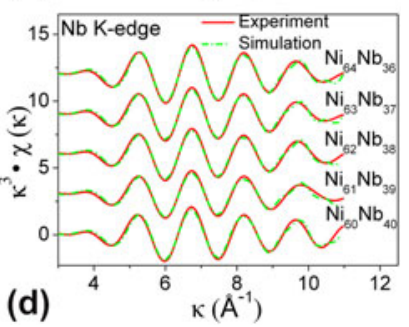

FIG. 2. (a) The RMC simulated three-dimensional atomic configuration of $\mathrm{Ni}_{62} \mathrm{Nb}_{38}$ (atomic configurations of other samples are not shown here), $\mathrm{Ni}$ and $\mathrm{Nb}$ atoms are shown in blue and yellow colors, respectively. The simulated curves and their corresponding experimental data for all five samples, including: (b) the structure factor, $S(Q)$, (c) Ni K-edge, and (d) Nb K-edge EXAFS spectra. The solid and dashed lines denote experimental and simulation data, respectively. The $k$ and $\chi(k)$ represent the photoelectron wave vector and the $k$-space EXAFS signal, respectively. (color online) 
form the microstructure in MGs. ${ }^{22,24,25}$ The clusters can be extracted from three-dimensional structural models obtained from the RMC simulation, and indexed according to their topological and geometrical features. By this method, all the VCs with $\mathrm{Ni}$ or $\mathrm{Nb}$ centers were obtained in the present work. The distributions of the major $\mathrm{Ni}$ and $\mathrm{Nb}$-centered VCs with various indexes are shown in Fig. 4.

In previous work, it suggested that besides the so-called ideal icosahedron (the $\langle 0,0,12,0\rangle$ cluster), other units (such as those indexed as $\langle 0,2,8,2\rangle,\langle 0,3,6,3\rangle$, $<0,1,10,2\rangle$, and so on) also are icosahedral or icosahedrallike clusters. ${ }^{32}$ As shown in Fig. 4, many $\mathrm{Ni}$ and $\mathrm{Nb}$ atoms in all the samples are prone to be centered in some icosahedrallike VCs, such as those indexed as $<0,2,8,2\rangle,\langle 0,3,6,3\rangle$,

TABLE II. The first-shell atomic distances $(R)$ and CNs around $\mathrm{Ni}$ and $\mathrm{Nb}$ atoms, respectively, which were obtained from the RMC simulation.

\begin{tabular}{lccccccccc}
\hline \hline & \multicolumn{3}{c}{$R(\AA) \pm 0.01$} & & \multicolumn{4}{c}{$\mathrm{CN} \pm 0.01$} \\
\cline { 2 - 4 } \cline { 8 - 9 } Samples & $\mathrm{Ni}-\mathrm{Ni}$ & $\mathrm{Ni}-\mathrm{Nb}$ & $\mathrm{Nb}-\mathrm{Nb}$ & & $\mathrm{Ni}-\mathrm{Ni}$ & $\mathrm{Ni}-\mathrm{Nb}$ & $\mathrm{Nb}-\mathrm{Ni}$ & $\mathrm{Nb}-\mathrm{Nb}$ \\
\hline $\mathrm{Ni}_{60} \mathrm{Nb}_{40}$ & 2.73 & 2.80 & 2.82 & & 7.28 & 5.55 & 8.33 & 4.87 \\
$\mathrm{Ni}_{61} \mathrm{Nb}_{39}$ & 2.73 & 2.79 & 2.82 & & 7.42 & 5.43 & 8.45 & 4.68 \\
$\mathrm{Ni}_{62} \mathrm{Nb}_{38}$ & 2.73 & 2.79 & 2.81 & & 7.63 & 5.27 & 8.59 & 4.67 \\
$\mathrm{Ni}_{63} \mathrm{Nb}_{37}$ & 2.72 & 2.78 & 2.82 & & 7.76 & 5.13 & 8.73 & 4.52 \\
$\mathrm{Ni}_{64} \mathrm{Nb}_{36}$ & 2.73 & 2.79 & 2.81 & & 7.86 & 5.05 & 8.96 & 4.31 \\
\hline \hline
\end{tabular}
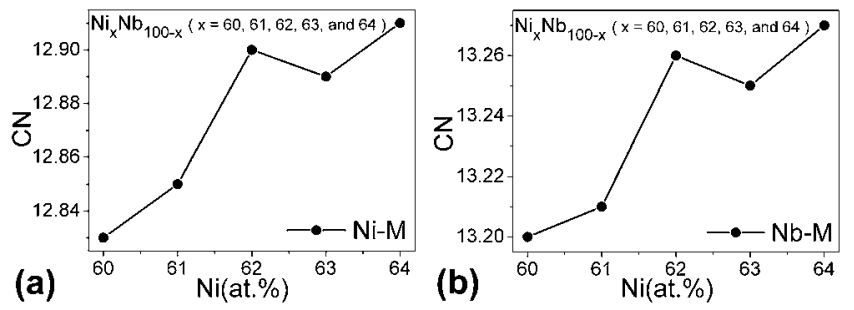

FIG. 3. The CNs around (a) $\mathrm{Ni}$ atoms and (b) $\mathrm{Nb}$ atoms. These $\mathrm{CN}$ values were obtained by calculating the number of the shell atoms in the Ni- or Nb-centered Voronoi-tessellated clusters. $\mathrm{M}$ denotes $\mathrm{Ni}$ and $\mathrm{Nb}$ atoms. and $<0,1,10,2>$. This is coincident with the widely accepted viewpoint that the icosahedral clusters are the preferred building blocks contributing to the formation of glassy alloys. ${ }^{41,42}$

Generally speaking, the distributions of $\mathrm{Ni}$ - or $\mathrm{Nb}$-centered VCs in these five samples are similar to each other. Nevertheless, the $\mathrm{Ni}_{62} \mathrm{Nb}_{38}$ MG has relatively high fractions of the VCs possessing relatively large $\mathrm{CNs}$ than other four alloys (such as Ni-centered VCs indexed as $<0,3,6,4\rangle$ and $<0,1,10,2\rangle$ with $\mathrm{CNs}$ of 13 , and $\mathrm{Nb}$-centered VCs indexed as $<0,3,6,4>$ with a $\mathrm{CN}$ of 13 and $<0,2,8,4>$ with a $\mathrm{CN}$ of 14$)$. This is in accordance with the deduced atom-level structural information that the total $\mathrm{CNs}$ around both $\mathrm{Ni}$ and $\mathrm{Nb}$ centers exhibit a local maximum at the $\mathrm{Ni}_{62} \mathrm{Nb}_{38}$ composition.

In our previous work studying the microstructures in $\mathrm{Cu}_{x} \mathrm{Zr}_{100-x}(x=61.8,63,64,64.5$, and 65.5 at. $\%) \mathrm{MGs}^{27}$ the total $\mathrm{CNs}$ around $\mathrm{Cu}$ or $\mathrm{Zr}$ atoms exhibit no local maximum at $\mathrm{Cu}_{64} \mathrm{Zr}_{32}$ composition (the pinpoint composition with local GFA maximum), and the fractions of VCs having relatively large $\mathrm{CNs}$ in $\mathrm{Cu}_{64} \mathrm{Zr}_{32}$ are not apparently higher than those of other MG compositions. It indicates that compared with the CuZr binary alloy system, there are more obvious structural discrepancy between the best glass former and its compositional neighbors in the selected $\mathrm{NiNb}$ system.

Recently, at the macroscopic scale, it revealed that the mass density strongly relates to the GFA in MG. ${ }^{43}$ On the other hand, at the microstructure scale, atomic dense packing also has been recognized as a key for the GFA in alloy systems. ${ }^{24,44,45}$ In the present work, a scheme was developed for calculating the atomic-level packing efficiencies $(\eta)$ inside the deduced $\mathrm{Ni}$ - and $\mathrm{Nb}$-centered VCs for all the samples, according to the equation,

$$
\eta=\frac{V_{\mathrm{a}}}{V_{\mathrm{u}}}
$$
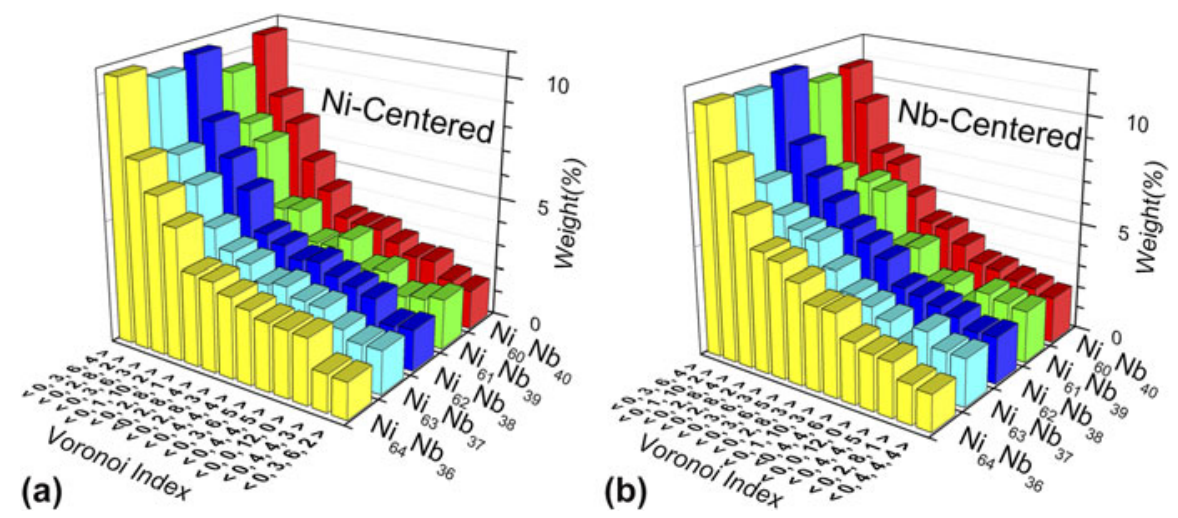

FIG. 4. Distribution of the major VCs, centered with (a) Ni and (b) Nb atoms. Note only VCs possessing weight over $2 \%$ were selected. (color online) 
where $V_{\mathrm{a}}$ and $V_{\mathrm{u}}$ are the atomic volume embedded in the clusters and the total volume of the clusters themselves, respectively. Each VC is formed by stacking a series of tetrahedra with a shared vertex, where the VC's center atom is sited. The $V_{\mathrm{u}}$ value can be obtained by summing the volumes of all the tetrahedra contained in the VCs. Because each atom embedded in the cluster is truncated by the edges, $V_{\mathrm{a}}$ should be calculated by summing up the volumes of all the truncated atoms.

In addition, the atomic-packing efficiencies $\left(\eta_{c}\right)$ in the hypothetical "ideal" crystal alloys were calculated for each selected $\mathrm{NiNb}$ composition by

$$
\begin{gathered}
\eta_{\mathrm{c}}=\sum_{i} C_{i} \eta_{i}, \\
\eta_{i}=\frac{\sum_{j} X_{j} V_{\mathrm{aj}}}{\sum_{j} X_{j} V_{\mathrm{uj}}},
\end{gathered}
$$

where $C_{i}$ and $\eta_{i}$ denote the concentration and atomicpacking efficiency of the $i$ th crystal component to form the "ideal" crystal in theory, respectively (in this work, the crystal components are $\mathrm{Ni}_{3} \mathrm{Nb}_{1}$ and $\mathrm{Ni}_{6} \mathrm{Nb}_{7}$ phases according to the compositions of samples). $X_{j}$ is the fraction of the $\mathrm{Ni}$ - or Nb-centered clusters with a center atom at the $j$ th Wykoff site (the method to extract clusters in crystal phases refers to our previous work $\left.{ }^{16}\right)$, whereas $V_{\text {aj }}$ and $V_{\mathrm{c} j}$ stand for the volume of the embedded atoms in the $j$ th type of clusters and the volume of the $j$ th type of clusters themselves, respectively.

The atomic-packing efficiencies $\left(\eta_{c}\right)$ in the hypothetical ideal crystal alloys are shown in Fig. 5. A linear relationship is found that the $\eta_{c}$ value monotonically increases with the $\mathrm{Ni}$ concentration. This indicates that the atomic-packing efficiency will increase when more small atoms replace the large ones in crystals. The calculated atomic-packing efficiencies of VCs for all the samples also are plotted in Fig. 5. The $\eta$ values of Ni-centered clusters are apparently smaller than those in crystal alloys, whereas the $\eta$ values are larger than their $\eta_{\mathrm{c}}$ counterparts in the Nb-centered clusters. In addition, it is worth noting that a local maximum of the atomic-packing efficiency for both the $\mathrm{Ni}$ - and $\mathrm{Nb}$-centered VCs is located at the $\mathrm{Ni}_{62} \mathrm{Nb}_{38}$ composition, which happens to be the best glass former in these selected compositions. This indicates that the atomic-packing efficiency strongly influences the GFA in alloys. If the atoms in these VCs possess the highest atomic-packing efficiency in the glassy state, the VCs will be very compact and stable, making them less likely to collapse or transform into the preferred crystal VCs during quenching, resulting in high GFA.

The differences between these two sets of packing efficiencies are magnified in Fig. 6. It is an interesting observation that a local maximum of the difference ratios
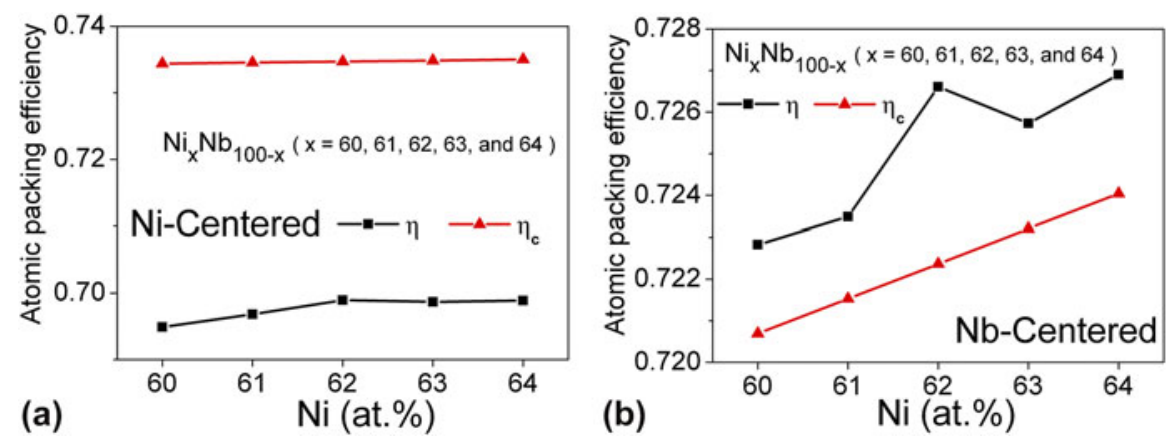

FIG. 5. The atomic-packing efficiencies, $\eta$, in five selected NiNb MGs and their corresponding values, $\eta_{\mathrm{c}}$, of the hypothetical ideal crystal alloys, for (a) Ni-centered and (b) Nb-centered clusters. (color online)
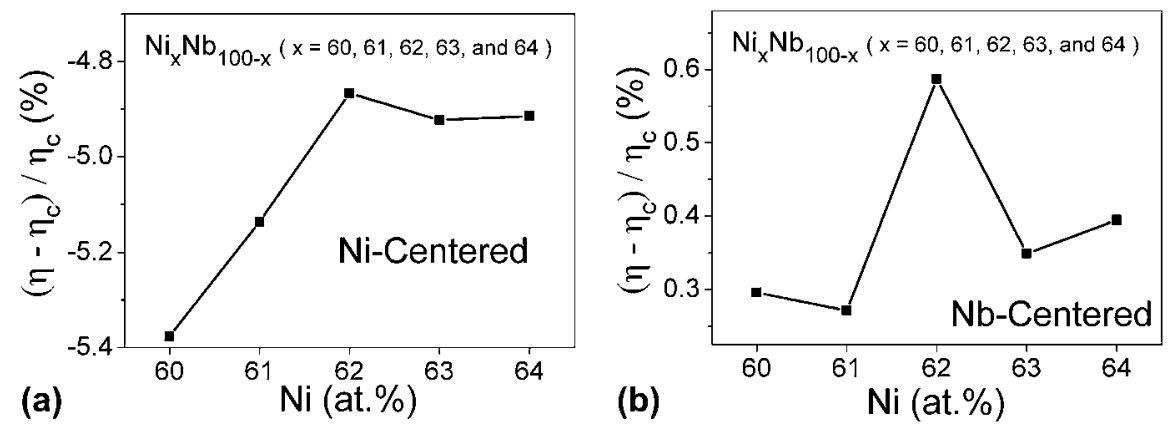

FIG. 6. The difference ratio between $\eta$ and $\eta_{c}$, i.e., $\left(\eta-\eta_{c}\right) / \eta_{c}$, for (a) Ni-centered and (b) Nb-centered clusters. 
appears in the $\mathrm{Nb}$-centered clusters of $\mathrm{Ni}_{62} \mathrm{Nb}_{38}$, i.e., the composition with the local GFA maximum. The $\mathrm{Ni}$ and $\mathrm{Nb}$ atoms should be regarded as the solvents and the solutes, respectively, considering their concentrations. This indicates that the solute-centered clusters are more compact and closed structural units to build the backbones of glass alloys than those centered with solvent atoms. The atomicpacking efficiency of the solute-centered clusters relates to the GFA that a relatively high packing efficiency has a one-to-one correspondence to the best glass former, resulting in the pinpoint-composition effect.

In previous work, it was proposed that the local structures closest to the spherical symmetry leads to the best GFA, ${ }^{46,47}$ which suggested that the regularity of clusters also may be a structural feature contributing to the GFA. It is acknowledged that the more regular a polyhedron is, the more compact it should be, such as the regular tetrahedron, the cube, and the sphere. Thus, it probably is the cluster-level regularity that determines the atomic-packing efficiency inside clusters, resulting in the structural influence on the glass formation in alloy systems.

To examine this scenario, the regularity of the VCs in all $\mathrm{NiNb}$ samples was investigated. The steradian parameter $(S)$ was adopted to denote the regularity of $\mathrm{VCs}$, which is the variance of the steradians of all the tetrahedra in VCs. $S$ can be calculated by

$$
S=\frac{n}{8 \pi^{2}(n-1)} \sum_{i<j}\left(S_{i}-S_{j}\right)^{2},
$$

Because each VC can be formed by topologically piling up a set of Delaunay tetrahedra sharing a vertex located by the center atom of the $\mathrm{VC},{ }^{36}$ thus, $S_{i}$ and $S_{j}$ stand for the steradians of the $i$ th and $j$ th tetrahedron contained in each VC, respectively, while $n$ is the total number of tetrahedra. It is apparent that for a regular tetrahedron, where all the steradians in the contained tetrahedra are the same, the value of $S$ should be zero.

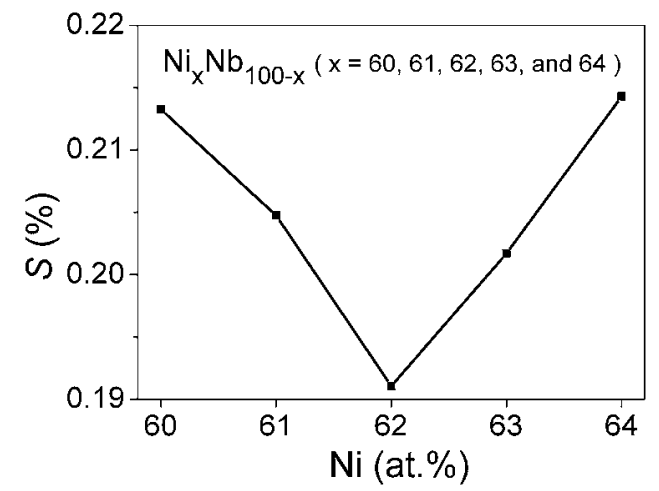

FIG. 7. The variance of the steradian, $S$, of all the tetrahedra forming the VCs in the selected five $\mathrm{NiNb}$ samples, which determines the regularity of VCs.
Therefore, if a tetrahedron has a smaller $S$ value, it has a higher degree of regularity.

The $S$ values denoting the regularity of VCs were calculated and shown in Fig. 7. It is observed that it is the $\mathrm{Ni}_{62} \mathrm{Nb}_{38}$ bulk MG that has the lowest $S$ value, which suggests that the VCs in this composition should possess the highest degree of regularity in these five samples. This indicates that the relatively high atomic-packing efficiency in $\mathrm{Ni}_{62} \mathrm{Nb}_{38}$ is due to its relatively regular VCs. Thus, besides the atomic-level efficiency, the cluster-level regularity should be another structural factor to determine the GFA.

\section{CONCLUSIONS}

In the present work, the pinpoint-composition effect on the GFA in binary alloys was investigated from the structural aspect. A series of NiNb binary glassy alloys were selected as the research prototypes that their microstructures were studied by calculations, simulations coupled with the synchrotron radiation-based XRD and EXAFS experimental data. It is revealed that some atomic- and cluster-level structural features influence the GFA. In particular, the highest atomic-level packing efficiency and the highest cluster-level regularity contribute to the formation of MG with the highest GFA. That is the structural origin of the pinpoint-composition effect on the GFA in binary alloy systems, which also may enhance understanding of the formation of various multicomponent alloys having the effect of microalloying on the GFA.

\section{ACKNOWLEDGMENTS}

The authors would like to thank HASYLAB in Germany, the Shanghai Synchrotron Radiation Facility in China, and the National Synchrotron Radiation Laboratory of China for the use of the advanced synchrotron radiation facilities. Financial support from the NUAA Research Funding (Grant No. NS2013057) also is gratefully acknowledged.

\section{REFERENCES}

1. W. Klement, R.H. Willens, and P. Duwez: Non-crystalline structure in solidified gold-silicon alloys. Nature 187, 869 (1960).

2. D. Turnbull: Under what conditions can a glass be formed? Contemp. Phys. 10, 473 (1969).

3. A.L. Greer: Confusion by design. Nature 366, 303 (1993).

4. A. Inoue: Stabilization of metallic supercooled liquid and bulk amorphous alloys. Acta Mater. 48, 279 (2000).

5. Z.P. Lu, H. Tan, Y. Li, and S.C. Ng: The correlation between reduced glass transition temperature and glass forming ability of bulk metallic glasses. Scr. Mater. 42, 667 (2000).

6. Z.P. Lu and C.T. Liu: Glass formation criterion for various glassforming systems. Phys. Rev. Lett. 91, 115505 (2003).

7. Z.Z. Yuan, S.L. Bao, Y. Lu, D.P. Zhang, and L. Yao: A new criterion for evaluating the glass-forming ability of bulk glass forming alloys. J. Alloys Compd. 459, 251 (2008). 
8. D.H. Xu, B. Lohwongwatana, G. Duan, W.L. Johnson, and C. Garland: Bulk metallic glass formation in binary $\mathrm{Cu}$-rich alloy series $-\mathrm{Cu}_{100-\mathrm{x}} \mathrm{Zr}_{\mathrm{x}}(\mathrm{x}=34,3638.2,40$ at.\%) and mechanical properties of bulk $\mathrm{Cu}_{64} \mathrm{Zr}_{36}$ glass. Acta Mater. 52, 2621 (2004).

9. L. Xia, W.H. Li, S.S. Fang, B.C. Wei, and Y.D. Dong: Binary Ni-Nb bulk metallic glasses. J. Appl. Phys. 99, 026103 (2006).

10. A. Inoue and W. Zhang: Formation, thermal stability and mechanical properties of $\mathrm{Cu}-\mathrm{Zr}$ and $\mathrm{Cu}-\mathrm{Hf}$ binary glassy alloy rods. Mater. Trans. 45, 584 (2004).

11. Z.W. Zhu, H.F. Zhang, W.S. Sun, B.Z. Ding, and Z.Q. Hu: Processing of bulk metallic glasses with high strength and large compressive plasticity in $\mathrm{Cu}_{50} \mathrm{Zr}_{50}$. Scr. Mater. 54, 1145 (2006).

12. L. Xia, S.T. Shan, D. Ding, and Y.D. Dong: Binary bulk metallic glass $\mathrm{Ni}_{62} \mathrm{Nb}_{38}$ with high compressive strength of $3100 \mathrm{MPa}$. Intermetallics 15, 1046 (2007).

13. N. Mattern, A. Schops, U. Kuhn, J. Acker, O. Khvostikova, and J. Eckert: Structural behavior of $\mathrm{Cu}_{\mathrm{x}} \mathrm{Zr}_{100-\mathrm{x}}$ metallic glass $(\mathrm{x}=35-70)$. J. Non-Cryst. Solids 354, 1054 (2008).

14. X.D. Wang, S. Yin, Q.P. Cao, J.Z. Jiang, H. Franz, and Z.H. Jin: Atomic structure of binary $\mathrm{Cu}(64.5) \mathrm{Zr}(35.5)$ bulk metallic glass. Appl. Phys. Lett. 92, 011902 (2008).

15. J. Basu, B.S. Murty, and S. Ranganathan: Glass forming ability: Miedema approach to $(\mathrm{Zr}, \mathrm{Ti}, \mathrm{Hf})-(\mathrm{Cu}, \mathrm{Ni})$ binary and ternary alloys. J. Alloys Compd. 465, 163 (2008).

16. L. Yang, J.H. Xia, Q. Wang, C. Dong, L.Y. Chen, X. Ou, J.F. Liu, J.Z. Jiang, K. Klementiev, K. Saksl, H. Franz, J.R. Schneider, and L. Gerward: Design of $\mathrm{Cu}_{8} \mathrm{Zr}_{5}$-based bulk metallic glasses. Appl. Phys. Lett. 88, 241913 (2006).

17. W.H. Wang: Roles of minor additions in formation and properties of bulk metallic glasses. Prog. Mater. Sci. 52, 540 (2007).

18. D.H. Xu, G. Duan, and W.L. Johnson: Unusual glass-forming ability of bulk amorphous alloys based on ordinary metal copper. Phys. Rev. Lett. 92, 245504 (2004).

19. W. Zhang, F. Jia, Q. Zhang, and A. Inoue: Effects of additional Ag on the thermal stability and glass-forming ability of $\mathrm{Cu}-\mathrm{Zr}$ binary glassy alloys. Mater. Sci. Eng., A 459, 330 (2007).

20. B. Li, Z.Y. Li, J.G. Xiong, L. Xing, D. Wang, and Y. Li: Laser welding of $\mathrm{Zr}_{45} \mathrm{Cu}_{48} \mathrm{~A}_{17}$ bulk glassy alloy. J. Alloys Compd. 413, 118 (2006).

21. L.Y. Chen, H.T. Hu, G.Q. Zhang, and J.Z. Jiang: Catching the Ni-based ternary metallic glasses with critical diameter up to $3 \mathrm{~mm}$ in $\mathrm{Ni}-\mathrm{Nb}-\mathrm{Zr}$ system. J. Alloys Compd. 443, 109 (2006).

22. J.D. Bernal: A geometrical approach to the structure of liquids. Nature 183, 141 (1959).

23. P.H. Gaskell: A new structural model for transition metal-metalloid glasses. Nature 276, 484 (1978).

24. D.B. Miracle: A structural model for metallic glasses. Nat. Mater. 3, 697, (2004).

25. H.W. Sheng, W.K. Luo, F.M. Alamgir, J.M. Bai, and E. Ma: Atomic packing and short-to-medium range order in metallic glasses. Nature 439, 419 (2006).

26. K.P. Tai, L.T. Wang, and B.X. Liu: Distinct atomic structures of the $\mathrm{Ni}-\mathrm{Nb}$ metallic glasses formed by ion beam mixing. J. Appl. Phys. 102, 124902 (2007).

27. L. Yang, G.Q. Guo, L.Y. Chen, C.L. Huang, T. Ge, D. Chen, P.K. Liaw, K. Saksl, Y. Ren, Q.S. Zeng, B. LaQua, F.G. Chen, and
J.Z. Jiang: Atomic-scale mechanisms of the glass-forming ability in metallic glasses. Phys. Rev. Lett. 109, 105502 (2012).

28. L. Yang, G.Q. Guo, G.Q. Zhang, and L.Y. Chen: Structural origin of the high glass-forming ability in Y-doped bulk metallic glasses. J. Mater. Res. 25, 1701 (2010).

29. http://www.pa.msu.edu/cmp/billinge-group/programs/PDFgetX/.

30. http://www.cells.es/Beamlines/CLAESS/software/viper.html.

31. L. Yang, S. Yin, X.D. Wang, Q.P. Cao, J.Z. Jiang, K. Saksl, and H. Franz: Atomic structure in $\mathrm{Zr}_{70} \mathrm{Ni}_{30}$ metallic glass. J. Appl. Phys. 102, 083512 (2007).

32. S.Y. Wang, M.J. Kramer, M. Xu, S. Wu, S.G. Hao, D.J. Sordelet, K.M. Ho, and C.Z. Wang: Experimental and ab initio molecular dynamics simulation studies of liquid $\mathrm{Al}_{60} \mathrm{Cu}_{40}$ alloy. Phys. Rev. $B$ 79, 144205 (2009).

33. R.L. McGreevy and L. Pusztai: Reverse Monte Carlo simulation: A new technique for the determination of disordered structures. Mol. Simul. 1, 359 (1988).

34. http://www.rmc-forum.org/Downloads/RMCA.

35. K. Saksl. P. Jovari, H. Franz, Q.S. Zeng, J.F. Liu, and J.Z. Jiang: Atomic structure of A189La6Ni5 metallic glass. J. Phys.: Condens. Matter 18, 7579 (2006).

36. http://www.ccl.net/cca/software/SOURCES/FORTRAN/allen-tildesleybook/f.35. shtml.

37. N.N. Medvedev: Algorithm for three-dimensional Voronoi polyhedra. J. Comput. Phys. 67, 223 (1986).

38. Q.S. Zeng, H.W. Sheng, Y. Ding, L. Wang, W.G. Yang, J.Z. Jiang, W.L. Mao, and H.K. Mao: Long-range topological order in metallic glass. Science 332, 1404 (2011).

39. L. Yang, G.Q. Guo, L.Y. Chen, S.H. Wei, J.Z. Jiang, and X.D. Wang: Atomic structure in Al-doped multicomponent bulk metallic glass. Scr. Mater. 63, 879 (2010).

40. D.B. Miracle, W.S. Sanders, and O.N. Senkov: The influence of efficient atomic packing on the constitution of metallic glasses. Philos. Mag. A 83, 2409 (2003).

41. J. Saida, M. Mstsushita, and A. Inoue: Direct observation of icosahedral cluster in Zr70Pd30 binary glassy alloy. Appl. Phys. Lett. 79, 412 (2001).

42. K. Saksl, H. Franz, P. Jovari, K. Klementiev, E. Welter, A. Ehnes, J. Saida, A. Inoue, and J.Z. Jiang: Evidence of icosahedral short-range order in $\mathrm{Zr}_{70} \mathrm{Cu}_{30}$ and $\mathrm{Zr}_{70} \mathrm{Cu}_{29} \mathrm{Pd}_{1}$ metallic glasses. Appl. Phys. Lett. 83, 3924 (2003).

43. Y. Li, Q. Guo, J.A. Kalb, and C.V. Thompson: Matching glass-forming ability with the density of the amorphous phase. Science 322, 1816 (2008).

44. A.L. Greer and E. Ma: Bulk metallic glasses: At the cutting edge of metals research. Mater. Res. Bull. 32, 611 (2007).

45. K.W. Park, J.I. Jang, M. Wakeda, Y. Shibutani, and J.C. Lee: Atomic packing density and its influence on the properties of $\mathrm{Cu}-\mathrm{Zr}$ amorphous alloys. Scr. Mater. 57, 805 (2007).

46. X.K. Xi, L.L. Li, B. Zhang, W.H. Wang, and Y. Wu: Correlation of atomic cluster symmetry and glass-forming ability of metallic glass. Phys. Rev. Lett. 99, 095501 (2007).

47. X.K. Xi, M.T. Sandor, Y.H. Liu, W.H. Wang, and Y. Wu: Structural changes induced by microalloying in $\mathrm{Cu}_{46} \mathrm{Zr}_{47-\mathrm{x}} \mathrm{A}_{17} \mathrm{Gd}_{\mathrm{x}}$ metallic glasses. Scr. Mater. 61, 967-969 (2009). 


\section{AUTHOR QUERY - jmr.2013.318}

1 Please provide the department (if any) for the affiliation "College of Materials Science and Technology." 